\title{
Study on steady-state thermal conduction with singularities in multi-
}

\section{material composites}

\author{
XF Hu ${ }^{\mathrm{a}}, \mathrm{HY} \mathrm{Gao}^{\mathrm{b}}$, WA Yao ${ }^{\mathrm{a}^{*}}$, ST Yang ${ }^{\mathrm{c}}$ \\ ${ }^{a}$ State Key Laboratory of Structural Analysis for Industrial Equipment, Dalian University of Technology, \\ Dalian 116024, China \\ ${ }^{b}$ Beijing Institute of Spacecraft Environment Engineering, Beijing, China \\ ${ }^{c}$ Department of Civil and Environmental Engineering, the University of Strathclyde, Glasgow, G1 IXJ, \\ United Kingdom \\ *Corresponding author: ywa@dlut.edu.cn
}

\begin{abstract}
Increasing demand in material and mechanical properties has led to production of complex composite structures. The composite structures, made of different materials, possess a variety of properties derived from each material. This has brought challenges in both analytical and numerical studies in thermal conduction which is of significant importance for thermoelastic problems. Therefore, a unified and effective approach would be desirable. The present study makes a first attempt to determining the analytical symplectic eigen solution for steady-state thermal conduction problem of multi-material crack. Based on the obtained symplectic eigen solution (including higher order expanding eigen solution terms), a new Symplectic Analytical Singular Element (SASE) for numerical modeling is constructed. It is concluded that composite structures composed of multi-material with complex geometric shapes can be modeled by the developed method, and the generalized flux intensity factors (GFIFs) can be solved accurately and efficiently.
\end{abstract}




\section{Nomenclature}

$\begin{array}{ll}A, B & \text { coefficients of the general solution of symplectic eigenvector } \\ \boldsymbol{A}, \boldsymbol{B} & \text { matrix composed of eigenvalues } \\ \boldsymbol{F} & \text { vector of coefficients of the general solution of symplectic eigenvector } \\ \boldsymbol{f}_{T}, \boldsymbol{f}_{r} & \text { vectors composed of eigenvectors' components } \\ \boldsymbol{H} & \text { Hamiltonian operator matrix } \\ k & \text { thermal conductivity } \\ \boldsymbol{K} & \text { stiffness matrix of the symplectic analytical singular element } \\ \boldsymbol{L} & \text { transform matrix } \\ M & \text { material } \\ P & \text { number of export node of the symplectic analytical singular element } \\ p & \text { parameter which is employed to define crack orientation } \\ q_{r}, q_{\theta} & \text { heat flux densities } \\ (r, \theta) & \text { polar coordinate system } \\ \boldsymbol{R} & \text { chain matrix relates the eigenvectors of two adjacent materials } \\ S_{r}, S_{\theta} & \text { symplectic dual variables } \\ \boldsymbol{t} & \text { vector of nodal temperature of the symplectic analytical singular element } \\ T & \text { temperature } \\ \boldsymbol{Z} & \text { unknown vector in symplectic solving system } \\ \alpha & \text { vertex angle of material } \\ \xi=1 n(r) & \text { transformation of the radial coordinate } \\ \rho & \text { radius of the proposed symplectic analytical singular element } \\ \nabla^{2} & \text { Laplacian operator } \\ \mu & \text { symplectic eigenvalue } \\ \mu^{i \text { ivariant }} & \text { singularity invariant } \\ \boldsymbol{\psi}(\theta) & \text { symplectic eigenvector } \\ \psi_{T}, \psi_{r} & \text { components of the symplectic eigenvector } \\ \psi_{J 1} & \text { Jordan form eigen vector } \\ \psi_{J} & \text { eigen solution formed by the Jordan form eigen vector } \\ \gamma & \text { coefficients of symplectic eigen expanding terms } \\ \Theta=0 & \text { characteristic equation of symplectic eigenvalue } \\ & \end{array}$




\title{
Study on steady-state thermal conduction with singularities in multi-
}

\section{material composites}

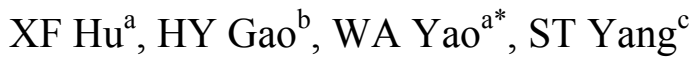 \\ ${ }^{a}$ State Key Laboratory of Structural Analysis for Industrial Equipment, Dalian University of Technology, Dalian \\ 116024, China \\ ${ }^{b}$ Beijing Institute of Spacecraft Environment Engineering, Beijing, China \\ ${ }^{c}$ Department of Civil and Environmental Engineering, the University of Strathclyde, Glasgow, G1 1XJ, United \\ Kingdom \\ ${ }^{*}$ Corresponding author: ywa@dlut.edu.cn
}

\begin{abstract}
Increasing demand in material and mechanical properties has led to production of complex composite structures. The composite structures, made of different materials, possess a variety of properties derived from each material. This has brought challenges in both analytical and numerical studies in thermal conduction which is of significant importance for thermoelastic problems. Therefore, a unified and effective approach would be desirable. The present study makes a first attempt to determining the analytical symplectic eigen solution for steady-state thermal conduction problem of multi-material cracks. Based on the obtained symplectic eigen solution (including higher order expanding eigen solution terms), a new Symplectic Analytical Singular Element (SASE) for numerical modeling is constructed. It is concluded that composite structures composed of multi-material with complex geometric shapes can be modeled by the developed method, and the generalized flux intensity factors (GFIFs) can be solved accurately and efficiently.
\end{abstract}

Keywords: symplectic dual approach, generalized flux intensity factor (GFIF), multi-material crack, steady-state thermal conduction 


\section{Introduction}

Composite materials and structures, such as thermal barrier coatings and electronic packages, are widely used in modern engineering applications as a result of increased demand on the properties of materials. In the construction of composite materials and structures, different materials are combined together to achieve enhanced characteristics that are not attainable by any single one of the materials used. However, cracks, such as defects, often arise between the material interfaces due to mismatch of different material properties during the fabrication. This could possibly lead to crack propagation under thermal loading. Therefore, analysis of heat conduction around multi-material crack tip is of high demand and great importance.

Heat conduction for two Dimensional (2D) homogeneous materials with cracks has been investigated by researchers, in which different boundary conditions on the crack surfaces were considered $^{[1,2]}$. Singularity of heat flux in the vicinity of crack tip in homogeneous material has been proven to exist with the order of $1 / 2^{[2]}$. Kuo studied bimaterial interface crack between two semiinfinite dissimilar media subject to uniform heat flow, and obtained the temperature distribution ${ }^{[3]}$. Chao and Shen investigated the interface crack between dissimilar media based on the Hilbert problem formulation and the exact solution was given for a single circular-arc $\operatorname{crack}^{[4]}$. Chao and Kuo studied heat conduction of curvilinear cracks in bounded dissimilar materials with heat source ${ }^{[5]}$. Chiu et al., and Tsai et al. investigated thermal conduction of an arbitrary oriented crack in functionally graded materials ${ }^{[6,7]}$. Buroni and his collaborators proposed a new complex-variable formalism for the analysis of $3 \mathrm{D}$ anisotropic heat transfer problems ${ }^{[8]}$. Marin proposed an invariant method of fundamental solutions to investigated 2D steady-state anisotropic heat conduction problems ${ }^{[9]}$.

For the structures with more general configuration, numerical methods such as extended finite element method $(\mathrm{XFEM})^{[10,11,12,13,14,15,16]}$, meshless method ${ }^{[17,18]}$ and boundary element method 
$(\mathrm{BEM})^{[19]}$ can be applied to get numerical solutions. Marin proposed a meshless method for the stable solution of direct and inverse problems associated with the two-dimensional Laplace equation in presence of noisy boundary data and boundary singularities, accurate numerical solutions can be obtained even when employing a small number of collocation points and sources ${ }^{[20]}$. The heat conduction problem in fins can be described by using the Helmholtz-type equation ${ }^{[21]}$. Marin studied the Helmholtz-type equation and proposed a meshless method for stable and accurate solution of direct and inverse problems in presence of boundary singularities; by using this method, the accuracy and convergence issues existing in conventional numerical methods were resolved ${ }^{[22,23]}$. Marin et al. applied BEM in solving the Helmholtz-type equation ${ }^{[24]}$. Mera et al. ${ }^{[25,26]}$ studied the Cauchy problem for steady-state anisotropic heat conduction problems by using BEM, while the standard BEM formulation was modified to take account of the stress singularity. It was demonstrated that the numerical algorithm was accurate, computational efficient and stable. Recently, the Singular Boundary Method (SBM) was applied to investigate the inverse anisotropic heat conduction problems ${ }^{[27]}$, heat conduction in nonhomogeneous materials ${ }^{[28]}$, and steady-state nonlinear heat conduction problem with temperaturedependent thermal conductivity ${ }^{[29]}$. Yosibash and his co-workers systematically investigated the steadystate thermal conduction problems with singularities and obtained numerical solutions of the generalized flux intensity factors (GFIF) based on post process operations in conjunction with $\mathrm{FEM}^{[30,31,32,33]}$. Yvonnet et al. investigated the Kapitza thermal resistance between two dissimilar materials by using 3D XFEM in which, the temperature jump across the interface were captured accurately with the aid of analytical solution ${ }^{[34]}$. Hosseini et al. studied crack propagation in functional graded materials subject to thermal and mechanical loadings by using XFEM, and reported that the implementation of crack tip enrichments could lead to substantial decrease of required number of degree of freedoms (DOFs), compared with standard FEM. Therefore, the solving efficiency for obtaining the same accuracy and 
convergence rate can be increased ${ }^{[35]}$. The extended isogeometric analysis was demonstrated to be suitable for the analysis of singularity problems ${ }^{[36,37]}$. Liu et al. studied functionally graded piezoelectric materials under thermal shock by the XFEM considering both heating and cooling shocks, the generalized dynamic intensity factors for thermal stresses and electrical displacements can be solved accurately by using the interaction integral ${ }^{[38]}$. Yu et al. investigated thermal buckling for functionally graded plates with internal defects using an extended isogeometric analysis, the trimmed NURBS surface to describe the geometrical structure with cutouts is no longer required as the internal discontinuity is independent on $\operatorname{mesh}^{[39]}$.

Recently, the symplectic dual approach for elasticity ${ }^{[40]}$ has emerged as a useful tool for the analysis of singularity problem. Analytical symplectic eigen solutions for bimaterial $\mathrm{crack}^{[41]}$, $\mathrm{crack}$ in pizeo-electric material ${ }^{[42,43]}$, and multi-material cracks under mechanical loading ${ }^{[44]}$ were obtained. Taking advantage of the existing analytical eigen solutions, a series of analytical singular elements were constructed for the numerical study of cracks ${ }^{[45]}$, fatigue crack growth ${ }^{[46]}$, cracks in Reissner plate $^{[47]}$ and Dugdale cohesive model based cracks ${ }^{[48]}$. The constructed analytical singular elements for each problem were found to be of high accuracy and efficiency. Leung et al. applied the symplectic dual approach to study steady-state thermal conduction problem of crack in homogeneous material, and the analytical symplectic eigen solution of the discussed problem was obtained ${ }^{[49]}$. In addition, Leung et al. studied thermal stress based on the obtained analytical symplectic eigen solution ${ }^{[50]}$. Zhou et al. proposed a numerical method based on the obtained symplectic eigen expansion to solve steady-state thermal conduction problems in bimaterial cracks ${ }^{[51]}$.

In light of the extensive literature review for the steady-state thermal conduction problem and other crack problems, the combination of near crack tip asymptotic solution and numerical method would bring many advantages in the analysis of composite materials with cracks ${ }^{[52,53]}$. The rich information of 
thermal variable fields around crack tip expressed in terms of analytical symplectic eigen solution can be applied to benefit the solving accuracy and efficiency of GFIFs (or known as eigen expanding coefficients). This will result in significant reduction in computing cost. Motivated by this purpose, the present study attempts, for the first time, to develop the analytical symplectic eigen solution for steadystate thermal conduction problem of multi-material crack and construct a new symplectic analytical singular element (SASE) based on the obtained analytical symplectic eigen solution.

The paper is organized as follows: the fundamental equations are summarized in section 2 , and the sub-coordinate system used in this study is defined in the same section. In section 3, the original problem is solved by using the symplectic dual approach and the analytical symplectic eigen solution of the discussed problem is obtained. Based on the obtained eigen solution, a SASE for steady-state thermal conduction problem of multi-material crack is constructed in section 4. The integration method of the proposed SASE as well as the calculation procedures of GFIFs are discussed in section 5. In section 6 , the singularity order of heat flux in multi-material crack is analyzed. In order to illustrate the present SASE, numerical examples are considered in section 7, which is followed by a conclusion in section 8 .

\section{Fundamental equations}

Considering a multi-material crack composed of $N(N \geq 1)$ different materials $M_{1}, M_{2}, \ldots$ as shown in Fig.1, the sub polar coordinate system $O C_{i} \theta_{i}$ is introduced to describe the $i$-th material $M_{i}$ with vertex angle $\alpha_{i}$. Thus the range of the angular coordinate for material $M_{i}$ is always $\theta_{i} \in\left[0, \alpha_{i}\right]$. For the steady-state thermal conduction problem, the relationship between the temperature and the heat flux densities in material $M_{i}$ can be specified by:

$$
q_{r, i}=-k_{i} \frac{\partial T_{i}}{\partial r}, \quad q_{\theta, i}=-\frac{k_{i}}{r} \frac{\partial T_{i}}{\partial \theta_{i}},(i=1,2,3, \ldots, N)
$$

where $T_{i}$ is temperature and $\left[q_{r, i}, q_{\theta, i}\right]$ is the vector of heat flux densities along each direction, and $k_{i}$ is the thermal conductivity. 
The governing equation neglecting the internal heat source can be specified by

$$
\nabla^{2} T_{i}=0,(i=1,2,3, \ldots, N)
$$

where $\nabla^{2}=\partial_{r}^{2}+(1 / r) \partial_{r}+\left(1 / r^{2}\right) \partial_{\theta}^{2}$ is the Laplacian operator in the polar coordinate system. The compatibility conditions at the interface between material $M_{i}$ and $M_{i+1}$ are specified by

$$
\begin{gathered}
\left.T_{i}\right|_{\theta_{i}=\alpha_{i}}=\left.T_{i+1}\right|_{\theta_{i+1}=0},(i=1,2,3, \ldots, N-1) \\
\left.q_{\theta, i}\right|_{\theta_{i}=\alpha_{i}}=\left.q_{\theta, i+1}\right|_{\theta_{i+1}=0},(i=1,2,3, \ldots, N-1)
\end{gathered}
$$

Noted that in the sub-coordinate systems $O C_{i} \theta_{i}$ and $O C_{i+1} \theta_{i+1}$, the angular coordinates of the interface between material $M_{i}$ and $M_{i+1}$ are $\alpha_{i}$ and 0 , respectively. The fundamental equations can be derived from the following equation of dissipation of quantity of heat written as:

$$
\delta\left\{\sum_{i=1}^{N} \int_{0}^{\alpha_{i}} \int_{0}^{\infty}\left(q_{r, i} \frac{\partial T_{i}}{\partial r}+\frac{q_{\theta, i}}{r} \frac{\partial T_{i}}{\partial \theta_{i}}+\frac{1}{2 k_{i}}\left(q_{r, i}^{2}+q_{\theta, i}^{2}\right)\right) r \mathrm{~d} r \mathrm{~d} \theta_{i}\right\}=0
$$

Typical boundary conditions at the crack surfaces are combinations of specific temperature and/or heat flux density, and the standard homogeneous boundary conditions can be summarized as

(i) prescribed temperature: $\left.\quad T_{1}\right|_{\theta_{1}=0}=0,\left.\quad T_{N}\right|_{\theta_{N}=\alpha_{N}}=0$

(ii) prescribed temperature and heat flux: $\left.\quad T_{1}\right|_{\theta_{1}=0}=0, \quad \partial T_{N} /\left.\partial \theta_{N}\right|_{\theta_{N}=\alpha_{N}}=0$

(iii) prescribed heat flux: $\quad \partial T_{1} /\left.\partial \theta_{1}\right|_{\theta_{1}=0}=0, \quad \partial T_{N} /\left.\partial \theta_{N}\right|_{\theta_{N}=\alpha_{N}}=0$

In the above denotations, the subscript ${ }_{i}$ " represents the $i$-th material, and it will be omitted hereinafter except where it may cause confusion. 


\section{Symplectic eigen expansion}

By introducing $\xi=\ln r, S_{r}=r q_{r}, S_{\theta}=r q_{\theta}$, the variational principle, i.e., Eq.(5), can be transformed into the equation as follows,

$$
\delta\left\{\sum_{i=1}^{N} \int_{0}^{\alpha_{i}} \int_{0}^{\infty}\left(S_{r, i} \frac{\partial T_{i}}{\partial \xi}+S_{\theta, i} \frac{\partial T_{i}}{\partial \theta_{i}}+\frac{1}{2 k_{i}}\left(S_{r, i}^{2}+S_{\theta, i}^{2}\right)\right) \mathrm{d} \xi \mathrm{d} \theta_{i}\right\}=0
$$

Making the variation with respect to $S_{\theta}$ gives

$$
S_{\theta}=-k \frac{\partial T}{\partial \theta}
$$

Substituting $S_{\theta}$ back into the variational principle to eliminate $S_{\theta}$ leads to

$$
\delta\left\{\sum_{i=1}^{N} \int_{0}^{\alpha_{i}} \int_{0}^{\infty}\left(S_{r, i} \frac{\partial T_{i}}{\partial \xi}+\frac{S_{r, i}^{2}}{2 k_{i}}-\frac{k_{i}}{2}\left(\frac{\partial T_{i}}{\partial \theta_{i}}\right)^{2}\right) \mathrm{d} \xi \mathrm{d} \theta_{i}\right\}=0
$$

Making the variations of Eq.(11) with respect to $T$ and $S_{r}$ respectively, the symplectic dual equation can be specified as follows,

$$
\left[\begin{array}{l}
\partial T / \partial \xi \\
\partial S_{r} / \partial \xi
\end{array}\right]=\left[\begin{array}{cc}
0 & -1 / k \\
k \partial^{2} / \partial \theta^{2} & 0
\end{array}\right]\left[\begin{array}{l}
T \\
S_{r}
\end{array}\right]
$$

In the symplectic dual method, $T$ is also recognized as the configuration variable while $S_{r}$ the dual variable. The above symplectic dual equation can also be expressed in the form of matrix as follows,

$$
Z^{2}=H Z
$$

where $\boldsymbol{Z}=\left[T, S_{r}\right]^{\mathrm{T}}$, and the dot '.' represents the partial differentiation with respect to $\xi$. By using the method of variable separation and assuming that the solution is in the form of

$$
Z=e^{\mu \xi} \psi(\theta)
$$

the original problem can be transformed into a symplectic eigenvalue problem, defined as follows, 


$$
\boldsymbol{H} \psi(\theta)=\mu \psi(\theta)
$$

where the eigenvalue $\mu$ and the corresponding eigenvector $\psi(\theta)=\left[\psi_{T}, \psi_{r}\right]^{\mathrm{T}}$ form the symplectic eigen pair (or termed as symplectic eigen solution). Depending on the boundary conditions on the crack surfaces, zero eigenvalue and the corresponding eigenvector may exist and they should be determined by solving $\boldsymbol{H} \boldsymbol{\psi}(\theta)=0$. For the three sets of typical boundary conditions in Eq.(6)-(8), zero eigenvalue only exist for the case with prescribed heat flux on the crack surfaces, e.g. Eq.(7). And the corresponding eigenvector can be specified by

$$
\psi^{(1)}=\left[\begin{array}{ll}
1 & 0
\end{array}\right]^{\mathrm{T}}
$$

It actually represents the steady temperature field with zero heat fluxes and temperature uniformly distributed everywhere. Besides, the Jordan form eigenvector $\boldsymbol{\psi}_{J 1}^{(1)}$ should satisfy $\boldsymbol{H} \boldsymbol{\psi}_{J 1}^{(1)}=\boldsymbol{\psi}^{(1)}$, and solving this equation gives $\psi_{J 1}^{(1)}=\left[\begin{array}{ll}0 & -k\end{array}\right]^{\mathrm{T}}$. The Jordan form eigenvector forms the corresponding eigen solution by

$$
\psi_{J}^{(1)}=\psi_{J 1}^{(1)}+\xi \psi^{(1)}=\left[\begin{array}{ll}
\xi & -k
\end{array}\right]^{\mathrm{T}}
$$

which represents a temperature filed with a center heat generation. It can be proven that the second grade Jordan form eigenvector does not exist and the Jordan chain breaks here. For nonzero eigensolutions, solving the above symplectic characteristic equation gives the general solution of the eigenvector

$$
\psi(\theta)=\left[\psi_{T}, \psi_{r}\right]^{\mathrm{T}}=\left[\begin{array}{cl}
\sin (\mu \theta) & \cos (\mu \theta) \\
-k \mu \sin (\mu \theta) & -k \mu \cos (\mu \theta)
\end{array}\right]\left[\begin{array}{l}
A \\
B
\end{array}\right]
$$

where $\boldsymbol{F}=[A, B]^{\mathrm{T}}$ is the vector of undetermined coefficients. Substituting the above eigenvector into the compatibility conditions at the interface between the material $M_{i}$ and $M_{i+1}$, Eq. (2) and Eq. (3) gives 


$$
\boldsymbol{F}_{i+1}=\boldsymbol{R}_{i} \boldsymbol{F}_{i}=\left[\begin{array}{cc}
k_{i} / k_{i+1} \cos \left(\mu \alpha_{i}\right) & -k_{i} / k_{i+1} \sin \left(\mu \alpha_{i}\right) \\
\sin \left(\mu \alpha_{i}\right) & \cos \left(\mu \alpha_{i}\right)
\end{array}\right]\left[\begin{array}{c}
A_{i} \\
B_{i}
\end{array}\right]
$$

The above equation relates the coefficient vectors $\boldsymbol{F}_{i}$ and $\boldsymbol{F}_{i+1}$ explicitly. Similarly, the coefficient vector $\boldsymbol{F}_{n}$ of the $n$-th material can be expressed by using $\boldsymbol{F}_{1}$ explicitly, and the eigenvector of the $n$-th material can be specified by

$$
\boldsymbol{\psi}_{n}=\left[\begin{array}{cc}
\sin (\mu \theta) & \cos (\mu \theta) \\
-k_{n} \mu \sin (\mu \theta) & -k_{n} \mu \cos (\mu \theta)
\end{array}\right] \prod_{i=n-1}^{1} \boldsymbol{R}_{i} \boldsymbol{F}_{1}
$$

The solution of the original problem can be given in the form of eigen expansion after solving all the symplectic eigen solutions:

$$
\boldsymbol{Z}=\sum_{j=1}^{\infty} \gamma^{(j)} e^{\xi \mu^{(j)}} \boldsymbol{\psi}^{(j)}(\theta)
$$

where the superscript " $(j) "$ represents the symplectic eigen expanding order, and $\gamma^{(j)}$ is the $j$-th eigen expanding coefficients. Following the definition of the generalized flux intensity factor (GFIF) by Ref.[32], the expanding coefficients $\gamma^{(j)}(j=1,2, \ldots \infty)$ are the GFIFs. Unlike the definition of stress intensity factors (SIFs) in linear elastic fracture mechanics (LEFM), the GFIFs include higher order symplectic eigen expanding coefficients beside the terms with singularity. The analytical solution can be obtained after solving all the unknown GFIFs.

It may be noted that the symplectic eigenvalues and the vector $\boldsymbol{F}_{1}$ are still unknown in present symplectic solving system, and should be determined by the boundary conditions at the two crack surfaces. Actually, the coefficients vector $\boldsymbol{F}_{1}$ depends only on the boundary conditions on $\theta_{1}=0$. 
Substituting the eigenvector of the first material into the boundary condition on $\theta_{1}=0$ leads to the nontrivial solution of $\boldsymbol{F}_{1}$, shown as follows,

$$
\begin{gathered}
\boldsymbol{F}_{1}=[1,0]^{\mathrm{T}}, \text { for }\left.T_{1}\right|_{\theta_{1}=0}=0 \\
\boldsymbol{F}_{1}=[0,1]^{\mathrm{T}}, \text { for }\left.\left(\partial T_{1} / \partial \theta\right)\right|_{\theta_{1}=0}=0
\end{gathered}
$$

The symplectic eigenvalues should be determined by the boundary condition on $\theta_{N}=\alpha_{N}$, by substituting the eigenvector into the boundary condition on $\theta_{N}=\alpha_{N}$, i.e.,

$$
\Theta(\mu)=0
$$

The value of the symplectic eigenvalues can be solved from the above equation. For some simple cases, the analytical solutions of the above equation can be obtained while for complex situations it can be solved numerically by using the Newton iteration method.

\section{Symplectic analytical singular element (SASE)}

In this section, a singular element with radius $\rho$ for steady-state thermal conduction problem of multimaterial crack as shown in Fig. 2 is constructed. In finite element modeling, the area around the crack tip should be occupied by this singular element while the other area of the structure employs conventional finite elements. The developed singular element is connected with the surrounding conventional elements through the "export nodes" which are distributed on the element's circumference as shown in Fig.2. The node indexes are arranged from 1 to $P(P \geq N$ in practical usage) as illustrated in Fig.2, and the number of nodes is not limited to a specific value; more export nodes will benefit the solving accuracy but will also increase the implementation complexity. The interior fields of the developed element are described by using the above obtained analytical symplectic eigen solutions; for this reason it is termed as "symplectic analytical singular element (SASE)".

Choosing the first $P$ terms from Eq.(21) as trial functions: 


$$
T^{*}=\sum_{j=1}^{P} \gamma^{(j)} e^{\xi \mu^{(j)}} \psi_{T}^{(j)}, S_{r}^{*}=\sum_{j=1}^{P} \gamma^{(j)} e^{\xi \mu^{(j)}} \psi_{r}^{(j)}
$$

The superscript "*" represents trial function to differentiate from the full symplectic eigen expansion, i.e., Eq.(21). However, other expanding terms are ignored which will cause errors in the results. In fact, the errors can be minimized when sufficient numbers of expanding terms are selected. Rewriting the trial functions in form of matrix results in:

$$
T^{*}=\boldsymbol{f}_{T}^{\mathrm{T}} \boldsymbol{A} \boldsymbol{\gamma}, \quad S_{r}^{*}=\boldsymbol{f}_{r}^{\mathrm{T}} \boldsymbol{A} \boldsymbol{\gamma}
$$

where $\gamma=\left[\gamma^{(1)}, \gamma^{(2)}, \ldots \gamma^{(P)}\right]^{\mathrm{T}}$ is the vector of unknown GFIFs (eigen expanding coefficients) and

$$
\begin{gathered}
\boldsymbol{A}=\operatorname{diag}\left(e^{\xi \mu^{(1)}}, e^{\xi \mu^{(2)}}, e^{\xi \mu^{(3)}} \ldots\right) \\
\boldsymbol{f}_{T}=\left[\psi_{T}^{(1)}, \psi_{T}^{(2)}, \ldots \psi_{T}^{(P)}\right]^{\mathrm{T}}, \boldsymbol{f}_{r}=\left[\psi_{r}^{(1)}, \psi_{r}^{(2)}, \ldots \psi_{r}^{(P)}\right]^{\mathrm{T}}
\end{gathered}
$$

Substituting the $i$-th export node's coordinates $\left(\rho, \theta_{i}\right)$ into the above expressions, the nodal temperature can be obtained easily, and the nodal temperature vector $\boldsymbol{t}=\left[T_{1}, T_{2}, \ldots T_{P}\right]^{\mathrm{T}}$ can be specified by

$$
\boldsymbol{t}=\boldsymbol{L B} \gamma
$$

where $\boldsymbol{B}=\operatorname{diag}\left(\rho^{\mu^{(1)}}, \rho^{\mu^{(2)}}, \rho^{\mu^{(3)}} \ldots\right)$ and $\boldsymbol{L}$ is the transform matrix specified by

$$
\boldsymbol{L}=\left[\begin{array}{c}
\psi_{T}^{(1)}\left(\theta_{1}\right), \psi_{T}^{(2)}\left(\theta_{1}\right), \ldots \psi_{T}^{(P)}\left(\theta_{1}\right) \\
\psi_{T}^{(1)}\left(\theta_{2}\right), \psi_{T}^{(2)}\left(\theta_{2}\right), \ldots \psi_{T}^{(P)}\left(\theta_{2}\right) \\
\ldots \\
\psi_{T}^{(1)}\left(\theta_{N}\right), \psi_{T}^{(2)}\left(\theta_{N}\right), \ldots \psi_{T}^{(P)}\left(\theta_{N}\right)
\end{array}\right]
$$

Meanwhile, the relationship between the unknown GFIFs (eigen expanding coefficients) and nodal temperature can be obtained and presented as follows,

$$
\gamma=\boldsymbol{B}^{-1} \boldsymbol{L}^{-1} \boldsymbol{t}
$$

Hence, the interior fields can be expressed by using the nodal temperature as follows,

$$
T^{*}=\boldsymbol{f}_{T}^{\mathrm{T}} \boldsymbol{A} \boldsymbol{B}^{-1} \boldsymbol{L}^{-1} \boldsymbol{t}, S_{r}^{*}=\boldsymbol{f}_{r}^{\mathrm{T}} \boldsymbol{A} \boldsymbol{B}^{-1} \boldsymbol{L}^{-1} \boldsymbol{t}
$$


The above formulas can be generally recognized as the "shape functions" in the frame of FEM although they are not standard polynomial FEM shape functions. Substituting the above equations into the variational principle represented by Eq.(11), and considering the trial functions satisfy the requirements of fundamental equations in the discussed domain and homogeneous boundary conditions on the crack surfaces, the variational principle can be simplified as follows,

$$
\delta\left\{\left.\sum_{i=1}^{N} \int_{0}^{\alpha_{i}\left(C_{i}\right)}\left[T^{*} S_{r}^{*}\right]\right|_{\xi=\ln \rho} \mathrm{d} \theta\right\}=0
$$

In addition, the stiffness matrix can be derived from the above variational principle:

$$
\boldsymbol{K}=\boldsymbol{L}^{-\mathrm{T}}\left(\sum_{i=1}^{N} \int_{0}^{\alpha_{i}\left(C_{i}\right)} \boldsymbol{f}_{T} \boldsymbol{f}_{r}^{\mathrm{T}} \mathrm{d} \theta\right) \boldsymbol{L}^{-1}
$$

It should be noted that the integration domain for each material is from 0 to $\alpha_{i}$ in the sub polar coordinate system $O C_{i} \theta_{i}$.

\section{Integration and GFIFs}

According to Eq. (34), it is interesting to find that the integration for the proposed 2D circular element is performed over its circumference (which is a 1D domain). Furthermore, since all the components in $\boldsymbol{f}_{T}$ and $\boldsymbol{f}_{r}$ are explicitly available, the integration can be achieved analytically.

By assembling the stiffness matrices of the SASE and standard elements into the global stiffness matrix, the original problem can be solved numerically. After solving the global equation, the nodal temperature of the SASE can be obtained, and the GFIFs can be calculated directly according to Eq.(31). The solving procedure is illustrated in Fig.3 for the convenience of readers. Unlike other methods, the complex post-processing is unnecessary in the proposed method. However, for crack propagation problem the proposed method still requires remeshing which is not required in the XFEM. Another 
possible drawback of the present method is that it is hard to extend the present method for threedimensional problems because it is very complex to get the corresponding eigen solutions.

\section{Singularity order (eigenvalues)}

In this section, a simple bimaterial crack is first analyzed followed by the analytical solution of eigenvalues and eigenvectors demonstrating the proposed method. Moreover, a special problem containing a five-material crack is investigated and the characteristic equation of eigenvalue is derived explicitly. It is interesting to find that a singularity invariant is proven to exist for a special case. Furthermore, other complex cases with similar multi-material structures are studied in which singularity invariants are also found existing.

Bimaterial crack: Considering a bimaterial interface crack problem, the boundary condition on crack surfaces can be presented as follows,

$$
\left.T\right|_{\theta_{1}=0}=0, \partial T /\left.\partial \theta\right|_{\theta_{2}=\pi}=0
$$

The eigenvalue satisfies $\tan (\pi \mu)= \pm \sqrt{k_{1} / k_{2}}$ and can be solved analytically which is shown as follows,

$$
\begin{gathered}
\psi_{1}=\left[\begin{array}{c}
1 \\
-k_{1} \mu
\end{array}\right] \sin (\mu \theta) \\
\psi_{2}=\left[\begin{array}{c}
1 / k_{2} \\
-\mu
\end{array}\right]\left(k_{2} \sin (\mu \pi) \cos (\mu \theta)+k_{1} \cos (\mu \pi) \sin (\mu \theta)\right) \\
S_{\theta, 1}=-k_{1} \mu \cos (\mu \theta), S_{\theta, 2}=k_{2} \mu \sin (\mu \theta) \sin (\mu \theta)-k_{1} \mu \cos (\mu \pi) \cos (\mu \theta)
\end{gathered}
$$

Specially, when $k_{1}=k_{2}$ the present solution is the same as that in Ref.[49]. This also verifies the proposed model. 
Singularity invariants: A special five-material crack is considered as shown in Fig.4, in which $\alpha_{2}=\alpha_{3}=\alpha_{4}=\pi / 2, \alpha_{1}=p \pi / 2,0<p<1$. The parameter $p$ is introduced to indicate the crack orientation. The material properties satisfy $k_{1} / k_{2} / k_{3} / k_{4} / k_{5}=1 / k / 1 / k / 1$. The boundary conditions on the crack surfaces are applied as follows:

$$
\partial T /\left.\partial \theta\right|_{\theta_{1}=0}=0, \partial T /\left.\partial \theta\right|_{\theta_{s}=\alpha_{5}}=0
$$

The characteristic equation of nonzero eigenvalue can be obtained and specified by

$$
\left[(k+1)^{2} \cos (\mu \pi)-(k-1)^{2}\right]\left\{\sin (p \mu \pi)[1-\cos (\mu \pi)]+\cos (p \mu \pi) \sin (\mu \pi)+\frac{(k+1)^{2}}{k^{2}-1} \sin (\mu \pi)\right\}=0
$$

The above equation is composed of two parts, the eigenvalues of each part can be solved separately. The eigenvalue of the first part is independent on $p$ (i.e. crack orientation in the specific material), and hence it is termed as singularity invariant which is given by

$$
\mu \pi=\arccos \left((k-1)^{2} /(k+1)^{2}\right), \quad 0<\mu<1
$$

In addition, the singularity invariant remains unchanged when replacing $k$ by $1 / k$. This implies that the singularity invariant always exist for arbitrary crack orientation in any material in Fig.4. The singularity invariant against crack orientation curve is shown in Fig.5. In this figure, only the $k>1$ part is shown because the solution remains unchanged when replacing $k$ by $1 / k$. The second part of Eq.(40) could still bring singularities which is dependent on the crack orientation, it is not listed here for the sake of simplicity.

Furthermore, the singularity invariants which are independent on crack orientation can also be found in similar structures shown in Fig.6. However, explicit forms of the eigenvalues of these complex structures can hardly be derived, whilst they should be solved numerically. It is found that two sets of singularity invariants exist in each case when $k=2$. The numerical results of singularity invariants are listed in 
Tab.1.

\section{Modeling of multi-material cracks using the SASE}

In this section, the steady-state thermal conduction problem in cracked structures is studied using the proposed SASE. The present solutions of GFIFs are compared with existing results and good agreements are found. Convergence studies on the number of the export nodes of the proposed SASE are conducted. After the verification, some complex structures are investigated to demonstrate the proposed method.

Cracked disc: A unit disc is considered and the meshed disc is shown in Fig.7, In the FE mesh, the crack tip area is occupied by the developed SASE with radius $\rho=0.5$ while the other area is meshed by using conventional isoparametric bilinear elements. The boundary conditions on the lower crack surface $\Gamma_{1}$ and upper surface $\Gamma_{2}$ are specified by:

$$
\partial T / \partial \theta=0, \text { on } \Gamma_{1}, \quad T=0, \text { on } \Gamma_{2}
$$

The boundary condition on the circular portion $\Gamma_{R}$ of the boundary of the disc is specified by

$$
\partial T / \partial r=y, \quad \text { on } \Gamma_{R}
$$

The symplectic eigenvalues can be solved analytically and specified by $\mu=(2 n-1) / 4, n=1,2,3, \ldots$. The approximate solution for this problem can be found in Ref.[32]

$$
T(r, \theta)=-1.35812 r^{1 / 4} \sin (\theta / 4)+0.970087 r^{3 / 4} \sin (3 \theta / 4)-0.452707 r^{5 / 4} \sin (5 \theta / 4)+O\left(r^{7 / 4}\right)
$$

The present results together with the results from Ref. [32] and Ref. [51] are listed in Tab.2, in which the convergence study on the number of export nodes of the proposed SASE is provided. Considering the results from Ref. [32] as benchmark, it is shown that when 17 export nodes are used the relative errors 
are less then $2.0 \%$. But when the number of export nodes increases to 31 , the relative errors are negligible. The contours of heat flux densities are shown in Fig.8, in which the heat fluxes distribution are very clear and strong concentrations in the vicinity of crack tip can easily be observed.

In addition to the single material cracked disc shown in Fig.7, a disc composed of four materials as illustrated in Fig.9 is considered. The boundary conditions are kept the same as the previous case. The thermal conductivities for each material are $k_{1} / k_{2} / k_{3} / k_{4}=1 / 2 / 3 / 4$. The eigenvalues should be solved numerically and the first few numerical results are listed in Tab.3.

The results of GFIFs are listed in Tab.4. It is found that with the increase of the selected export nodes the present solutions trend to converge, and the solutions are stable when 31 export nodes are selected. The contours of heat flux densities are shown Fig.10. Again, the gradients of the heat flux densities are very clear. It is interesting to find that $q_{\theta}$ is continuing over the domain but $q_{r}$ is not, and the material interfaces are the dividing lines of the distribution of $q_{r}$. Actually this is in line with the compatibility condition on the material interfaces. According to the present numerical examination, it is found that 31 export nodes can ensure the solving accuracy; so in the following discussions 31 export nodes of the SASE are used.

Double edge crack: A bimaterial $W \times W$ rectangular plate containing cracks on two sides is employed to demonstrate the application of the developed method. The geometry and mesh of the plate are shown in Fig.11. The length of the cracks is $a$ and $a / W=1 / 10$. The temperature on the upper surfaces of the cracks is zero while the lower surfaces of the cracks are insulated. Moreover, the left and right sides of the plate are also insulated. The temperatures on the lower and upper sides of the plate are $T_{1}$ and $T_{2}$, respectively. The sketch of finite element mesh is shown in Fig.11, only half of the specimen is actually calculated due to geometrical symmetry. The crack tip area is occupied by the SASE with radius 
$\rho=0.5 a$ while the other area is meshed using conventional isoparametric bilinear elements. The eigenvalue for this case can be solved analytically and specified by $\mu \pi=\arctan \left( \pm \sqrt{k_{1} / k_{2}}\right)$. In this example $T_{1}=100{ }^{\circ} \mathrm{C}$ is chosen and the numerical results of GFIFs with the variation of $T_{2}$ are listed in Tab.5. It is observed that the values of $\gamma_{1}, \gamma_{2}$ and $\gamma_{4}$ increase monotonically with $T_{2}$, while the values of $\gamma_{3}$ and $\gamma_{5}$ decrease with $T_{2}$.

Four-material edge crack: A $W \times 2 W$ rectangular plate made of four different materials and containing an edge crack is shown in Fig.12. The temperature on the upper surface of the crack is zero while the lower surface of the crack is insulated. The left and right sides of the plate are also insulated. The temperatures on the lower and upper sides of the plate are $T_{1}$ and $T_{2}$, respectively. The sketch of finite element mesh is also shown in Fig.12. The thermal conductivities are $k_{1} / k_{2} / k_{3} / k_{4}=1 / 2 / 3 / 4$ and the numerical results of eigenvalues can be found in Tab.3. The crack tip area is occupied by the SASE while the other area is meshed using conventional isoparametric bilinear elements. In this example $T_{1}=100{ }^{\circ} \mathrm{C}$ is chosen and the numerical results of GFIFs with the variation of $T_{2}$ are listed in Tab.6. It is observed that the values of $\gamma_{1}, \gamma_{2}$ and $\gamma_{4}$ increase monotonically with $T_{2}$, while the values of $\gamma_{3}$ and $\gamma_{5}$ decrease with $T_{2}$.

\section{Conclusion}

In this study, the steady-state thermal conduction problem with singularities of multi-material composites is investigated systematically. A unified procedure based on symplectic dual approach is proposed both for the analytical heat flux singularity study and the numerical finite element modeling study of the problem. The general form of the eigen expansion for multi-material crack are first derived 
in this study. In addition, a symplectic analytical singular element (SASE) is proposed and numerical solutions of generalized flux intensity factors (GFIFs) can be solved. Mesh refinement around crack tips are not necessary and the solving efficiency is therefore improved. For the multi-material cracks with special configurations and material combinations, an interesting singularity invariant which is independent on crack orientation is proven to exist. Numerical studies on some typical cracked multimaterial structures are given to demonstrate the application of the proposed SASE. Convergence studies have shown that in the proposed SASE 31 nodes are required to ensure the solving accuracy. The developed method can be further extended for thermal conduction problem in anisotropic materials in which research is being carried out.

\section{Acknowledgements}

The supports of the National Natural Science Foundation of China (No.11502045, and No. 11372065), the Fundamental Research Funds for the Central Universities (DUT15RC(3)029) are gratefully acknowledged.

\section{References}

[1]. Sih GC. Heat conduction in the infinite medium with lines of discontinuities. Journal of Heat Transfer, 1965, 87: 293298.

[2]. Kondrashov NG, Ryvkin VB. Isolation of the singularity in the solution of a heat conduction problem. Journal of Engineering Physics, 1967, 12: 47-50.

[3]. Kuo AY. Interface crack between two dissimilar half spaces subjected to a uniform heat flow at infinity-open crack. Journal of Applied Mechanics, 1990, 57: 359-364.

[4]. Chao CK, Shen MH. Thermal problem of curvilinear cracks in bonded dissimilar materials. Journal of Applied Physics, 1993, 73(11): 7129-7137.

[5]. Chao CK, Kuo LY. Thermal problem of curvilinear cracks in bonded dissimilar materials with a point heat source. International Journal of Heat and Mass Transfer, 1993, 36: 4085-4093.

[6]. Chiu TC, Tsai SW, and Chue CH, Heat conduction in a functionally graded medium with an arbitrarily oriented crack, International Journal of Heat and Mass Transfer, 2013, 67:514-522.

[7]. Tsai SW, Chiu TC, Chue CH, Temperature distribution and heat flow around a crack of arbitrary orientation in a functionally graded medium, Journal of Engineering Mathematics, 2014, 87:123-137.

[8]. Buroni FC, Marczk RJ, Denda M, Saez A. A formalism for anisotropic heat transfer phenomena: Foundations and Green's functions. International Journal of Heat and Mass Transfer, 2014, 75: 399-409.

[9]. Marin L. An invariant method of fundamental solutions for two-dimensional steady-state anisotropic heat conduction problems. International Journal of Heat and Mass Transfer, 2016, 94: 449-464. 
[10]. Yu TT, Bui TQ, Liu P, Zhang CZ, Hirose S. Interfacial dynamic impermeable cracks analysis in dissimilar piezoelectric materials under coupled electromechanical loading with the extended finite element method. International Journal of Solids and Structures, 2015, 67: 205-218.

[11]. Liu P, Bui TQ, Zhu D, Yu TT, Wang JW, Yin SH, Hirose S. Buckling failure analysis of cracked functionally graded plates by a stabilized discrete shear gap extended 3-node triangular plate element. Composites Part B: Engineering, 2015, 77: 179-193.

[12] Kang ZY, Bui TQ, Nguyen DD, Saitoh T, Hirose S. An extended consecutive-interpolation quadrilateral element (XCQ4) applied to linear elastic fracture mechanics. Acta Mechanica, 2015, 226: 3991-4015.

[13] Zhang XD, Bui TQ. A fictitious crack XFEM with two new solution algorithms for cohesive crack growth modeling in concrete structures. Engineering Computations, 2015, 32: 473-497.

[14]. Bui TQ, Zhang CZ. Analysis of generalized dynamic intensity factors of cracked magnetoelectroelastic solids by XFEM. Finite Elements in Analysis and Design, 2013, 69: 19-36.

[15]. Sharma K, Bui TQ, Zhang CZ, Bhargava RR. Analysis of a subinterface crack in piezoelectric bimaterials with the extended finite element method. Engineering Fracture Mechanics, 2013, 104: 114-139.

[16]. Bui TQ, Zhang CZ. Extended finite element simulation of stationary dynamic cracks in piezoelectric solids under impact loading. Computational Materials Science, 2012, 62: 243-257.

[17]. Tanaka S, Suzuki H, Sadamoto S, Imachi M, Bui TQ. Analysis of cracked shear deformable plates by an effective meshfree plate formulation. Engineering Fracture Mechanics, 2015, 144: 142-157.

[18]. Nguyen NT, Bui TQ, Zhang CZ, Truong TT. Crack growth modeling in elastic solids by the extended meshfree Galerkin radial point interpolation method. Engineering Analysis with Boundary Elements, 2014, 44: 87-97.

[19]. Lei J, Zhang CZ, Bui TQ. Transient dynamic interface crack analysis in magnetoelectroelastic bi-materials by a timedomain BEM. European Journal of Mechanics-A/Solids, 2015, 49: 146-157.

[20]. Marin L. Stable MFS solution to singular direct and inverse problems associated with the Laplace equation subjected to noisy data. CMES: Computer Modeling in Engineering \& Sciences, 2008, 37: 203-242.

[21]. Wood AS, Tupholme GE, Bhatti MIH, Heggs PJ. Steady-state heat transfer through extended plane surfaces. International Communications in Heat and Mass Transfer, 1995, 22: 99-109.

[22]. Marin L. A meshless method for the stable solution of singular inverse problems for two-dimensional Helmholtz-type equations. Engineering Analysis with Boundary Elements, 2010, 34: 274-288.

[23]. Marin L. Treatment of singularities in the method of fundamental solutions for two-dimensional Helmholtz-type equations. Applied Mathematical Modelling, 2010, 34: 1615-1633.

[24] Marin L, Lesnic D, Mantič V. Treatment of singularities in Helmholtz-type equations using the boundary element method. Journal of Sound and Vibration, 2004, 278: 39-62.

[25]. Mera NS, Elliott L, Ingham DB, Lesnic D. Singularities in anisotropic steady-state heat conduction using a boundary element method. International Journal for Numerical Methods in Engineering, 2002, 53: 2413-2427.

[26]. Mera NS, Elliott L, Ingham DB, Lesnic D. An iterative algorithm for singular Cauchy problems for the steady state anisotropic heat conduction equation. Engineering Analysis with Boundary Elements, 2002, 26: 157-168.

[27]. Gu Y, Chen W, Fu ZJ. Singular boundary method for inverse heat conduction problems in general anisotropic media. Inverse Problems in Science and Engineering, 2014, 22: 889-909.

[28]. Wei X, Chen W, Chen B, Sun L. Singular boundary method for heat conduction problems with certain spatially varying conductivity. Computers \& Mathematics with Applications, 2015, 69: 206-222.

[29]. Mierzwiczak M, Chen W, Fu ZJ. The singular boundary method for steady-state nonlinear heat conduction problem with temperature-dependent thermal conductivity. International Journal of Heat and Mass Transfer, 2015, 91: 205-217.

[30]. Yosibash Z, Szabo B, Numerical analysis of singularities in two dimensions Part 1: Computation of Eigenpairs, International Journal of Numerical Methods for Engineering, 1995, 38:2055-2082.

[31]. Omer N, Yosibash Z, Costabel M, Dauge M. Edge flux intensity functions in polyhedral domains and their extraction by a quasidual function method, International Journal of Fracture, 2004, 29:97-130.

[32]. Yosibash Z. Singularities in elliptic boundary value problems and elasticity and their connection with failure initiation. Springer, 2012.

[33]. Shannon S, Yosibash Z, Dauge M, Costabel M. Extracting generalized edge flux intensity functions with the quasidual function method along circular 3-D edges, International Journal of Fracture, 2013, 181:25-50.

[34]. Yvonnet J, He QC, Zhu QZ, Shao JF. A general and efficient computational procedure for modelling the Kapitza thermal resistance based on XFEM. Computational Materials Science. 2011, 50: 1220-1224.

[35]. Hosseini SS, Bayesteh H, Mohammadi S. Thermo-mechanical XFEM crack propagation analysis of functionally graded materials. Materials Science and Engineering: A, 2013, 561: 285-302. 
[36]. Bui TQ. Extended isogeometric dynamic and static fracture analysis for cracks in piezoelectric materials using NURBS. Computer Methods in Applied Mechanics and Engineering, 2015, 295: 470-509.

[37]. Bui TQ, Hirose S, Zhang CZ, Rabczuk T, Wu CT, Saitoh T, Lei J. Extended isogeometric analysis for dynamic fracture in multiphase piezoelectric/piezomagnetic composites. Mechanics of Materials, 2016, 97: 135-163.

[38]. Liu P, Yu T, Bui TQ, Zhang CZ, Xu YP, Lim CW. Transient thermal shock fracture analysis of functionally graded piezoelectric materials by the extended finite element method. International Journal of Solids and Structures, 2014, 51: 2167-2182.

[39] Yu TT, Bui TQ, Yin SH, Doan DH, Wu CT, Do TV, Tanaka S, On the thermal buckling analysis of functionally graded plates with internal defects using extended isogeometric analysis. Composite Structures, 2016, 136: 684-695.

[40]. Yao WA, Zhong WX, Lim CW. Symplectic elasticity. Singapore: World scientific, 2009.

[41]. Hu XF, Yao WA. A novel singular finite element on mixed-mode bimaterial interfacial cracks with arbitrary crack surface tractions. International Journal of Fracture, 2011, 172: 41-52.

[42]. Wang JS, Qin QH. Symplectic model for piezoelectric wedges and its application in analysis of electroelastic singularities. Philosophical Magazine, 2007, 87: 225-251.

[43]. Xu CH, Zhou ZH, Xu XS, Leung AYT. Electroelastic singularities and intensity factors for an interface crack in piezoelectric-elastic bimaterials. Applied Mathematical Modelling, 2015, 39: 2721-2739.

[44]. Zhang HW, Zhong WX. Hamiltonian principle based stress singularity analysis near crack corners of multi-material junctions. International Journal of Solids and Structures, 2003, 40: 493-510.

[45]. Yao WA, Hu XF. A novel singular finite element of mixed-mode crack problems with arbitrary crack tractions. Mechanics Research Communications, 2011, 38: 170-175.

[46]. Hu XF, Yao WA. A new enriched finite element for fatigue crack growth. International Journal of Fatigue, 2013, 48: 247-256.

[47]. Yao WA, Zhang ZJ, Hu XF. A singular element for Reissner plate bending problem with V-shaped notches. Theoretical and Applied Fracture Mechanics, 2014, 74: 143-156.

[48]. Yao WA, Hu XF. A Novel Singular Finite Element on Mixed-Mode Dugdale Model Based Crack. Journal of Engineering Materials and Technology, 2012, 134: 021003.

[49]. Leung AYT, Xu XS, Zhou ZH. Hamiltonian approach to analytical thermal stress intensity factors—Part 1: Thermal Intensity Factor. Journal of Thermal Stresses, 2010, 33: 262-278.

[50]. Leung AYT, Xu XS, Zhou ZH. Hamiltonian Approach to Analytical Thermal Stress Intensity Factors-Part 2 Thermal Stress Intensity Factor. Journal of Thermal Stresses, 2010, 33: 279-301.

[51]. Zhou ZH, Xu CH, Xu XS, Leung AYT. Finite-Element Discretized Symplectic Method for Steady-State Heat Conduction with Singularities in Composite Structures. Numerical Heat Transfer, Part B: Fundamentals, 2015, 67: 302-319.

[52]. Liu XY, Xiao QZ, Karihaloo BL. XFEM for direct evaluation of mixed mode SIFs in homogeneous and bi-materials. International Journal for Numerical Methods in Engineering, 2004, 59:1103-1118.

[53]. Xiao QZ, Karihaloo BL. Improving the accuracy of XFEM crack tip fields using higher order quadrature and statically admissible stress recovery. International Journal for Numerical Methods in Engineering, 2006, 66:1378-1410. 


\section{Captions of figures and tables}

Fig.1 Multi-material crack and the sub-coordinate system

Fig.2 Configuration of the symplectic analytical singular element(SASE) for multi-material crack

Fig.3 Solving procedure of the SASE based FE modelling

Fig.4 A special five material crack

Fig.5 The curve of the heat flux singularity invariant

Fig.6 Multi-material crack with similar structures

Fig.7 A cracked disc and the FE mesh with the symplectic analytical singular element(SASE)

Fig.8 Contours of heat flux densities around the tip of the crack shown in Fig.7.

Fig.9 Configuration of a four material disc containing a crack

Fig.10 Contours of heat flux densities around the four-material crack tip.

Fig.11 A double edge crack in a bimaterial rectangular plate and the FE mesh

Fig.12 A four material edge crack in a rectangular plate and the FE mesh

Tab.1 Singularity invariants of the multi-material crack

Tab.2 Generalized flux intensity factors(GFIFs) of the cracked single material disc

Tab.3 Numerical solutions of the first few symplectic eigenvalues the four material crack

Tab.4 Generalized flux intensity factors(GFIFs) of the cracked four-material disc

Tab.5 Generalized flux intensity factors(GFIFs) of the double edge cracked bimaterial plate

Tab.6 Generalized flux intensity factors(GFIFs) of the four material edge crack 


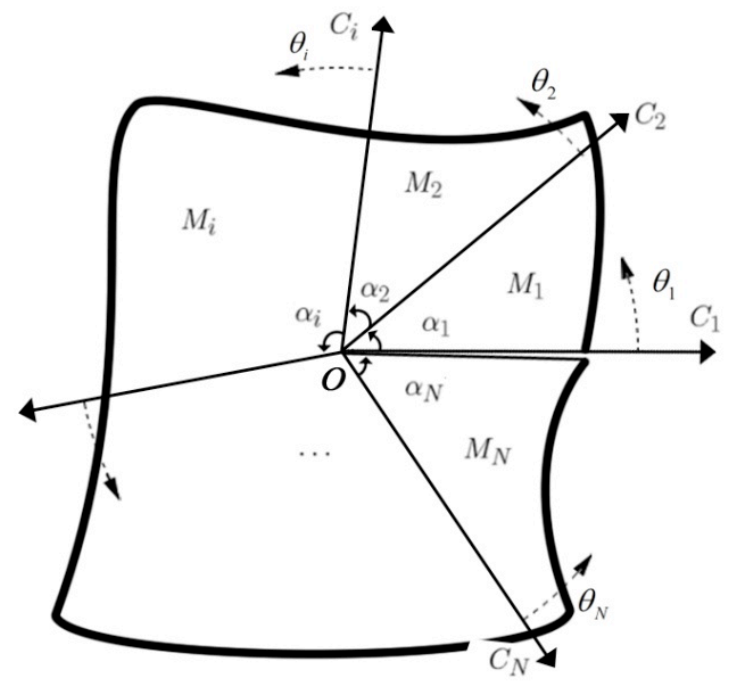

Fig.1 Multi-material crack and the sub-coordinate system

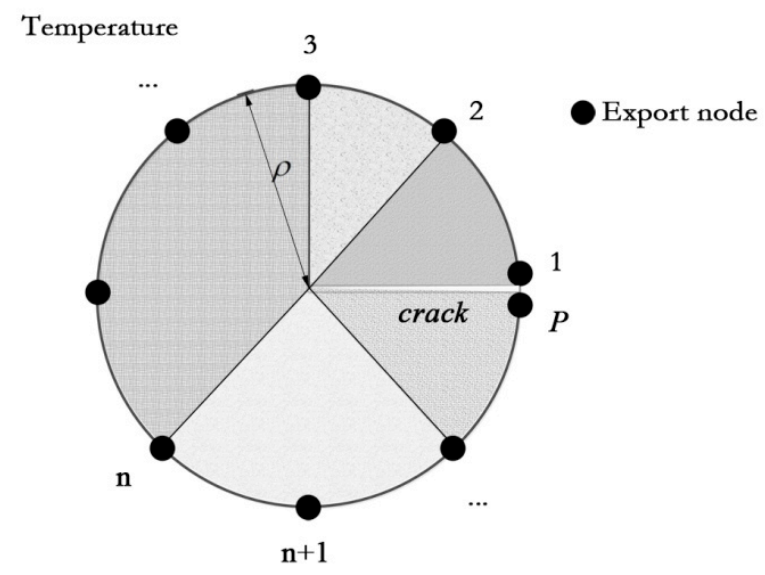

Fig.2 Configuration of the symplectic analytical singular element(SASE) for multi-material crack 


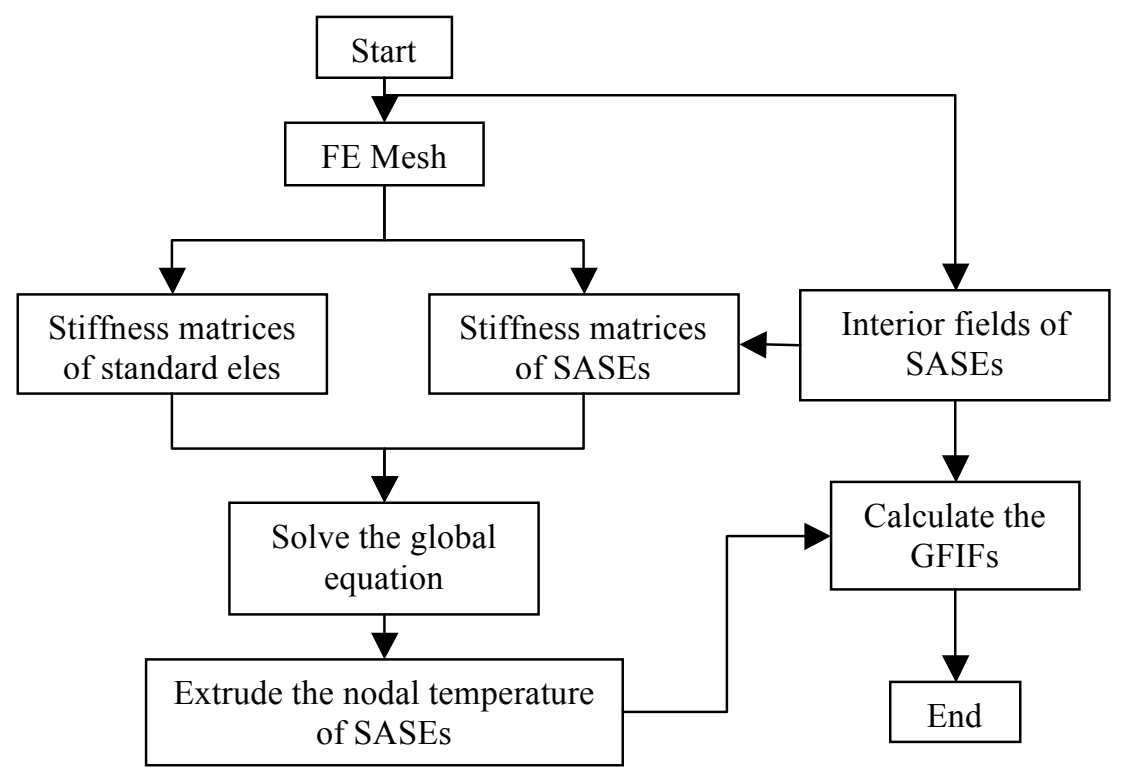

Fig.3 Solving procedure of the SASE based FE modelling

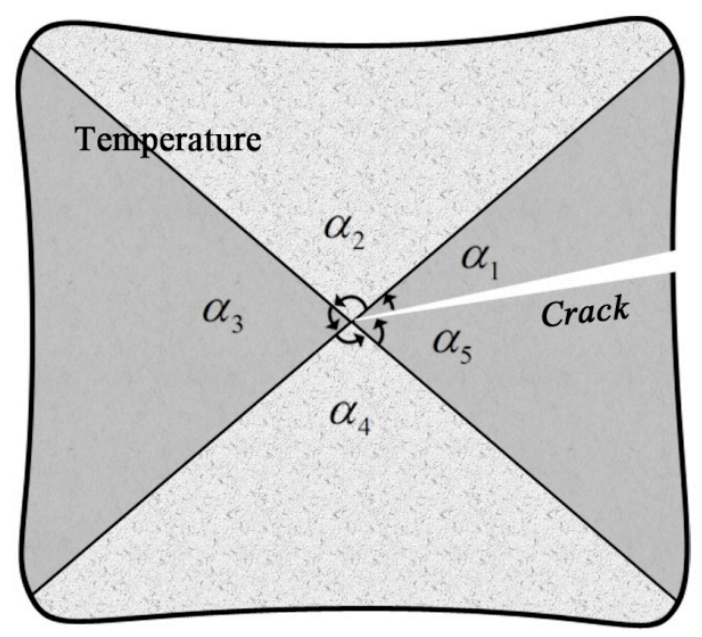

Fig.4 A special five material crack 


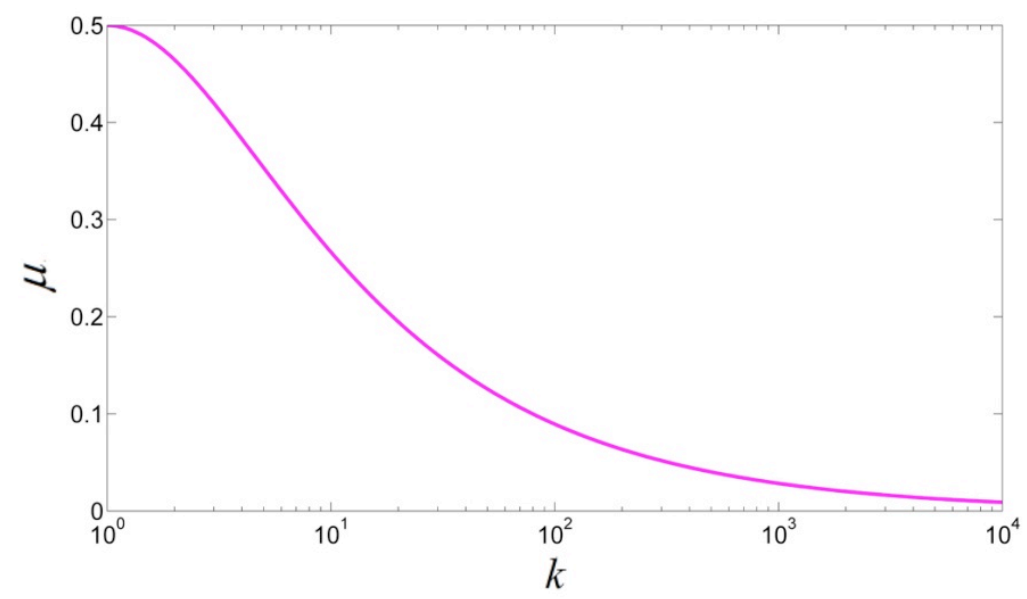

Fig.5 The curve of the heat flux singularity invariant

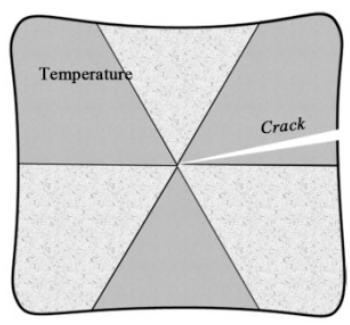

(a) 7 material

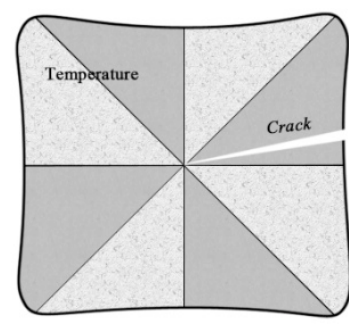

(b) 9 material

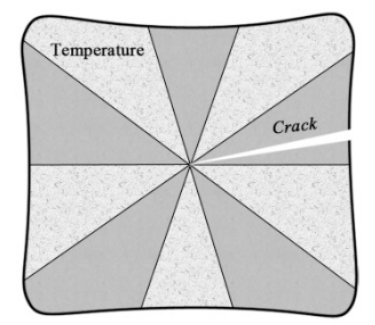

(c) 11 material

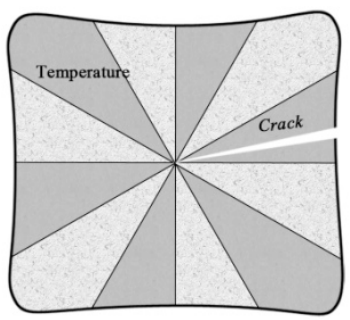

(d) 13 material

Fig.6 Multi-material crack with similar structures
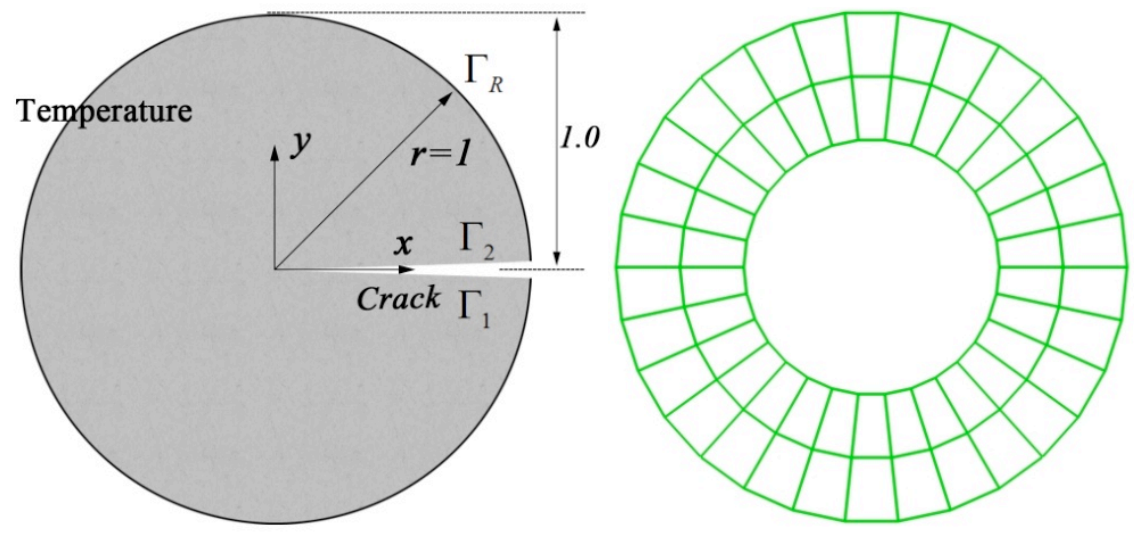

Fig.7 A cracked disc and the FE mesh with the symplectic analytical singular element(SASE) 


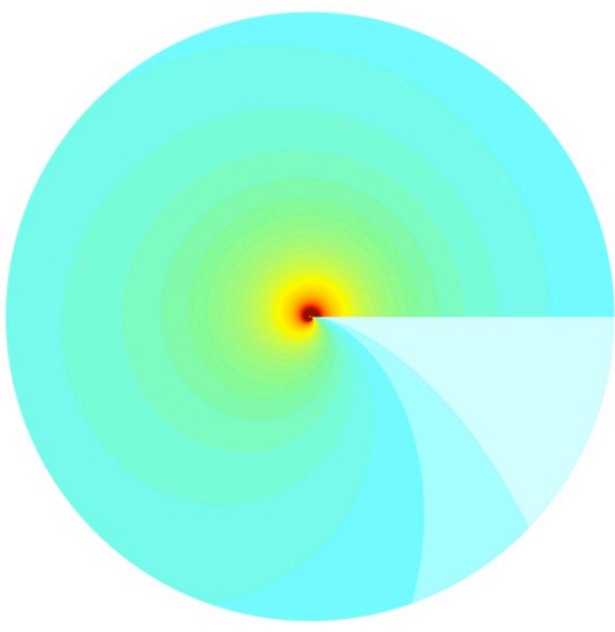

(a) $q_{\theta}$

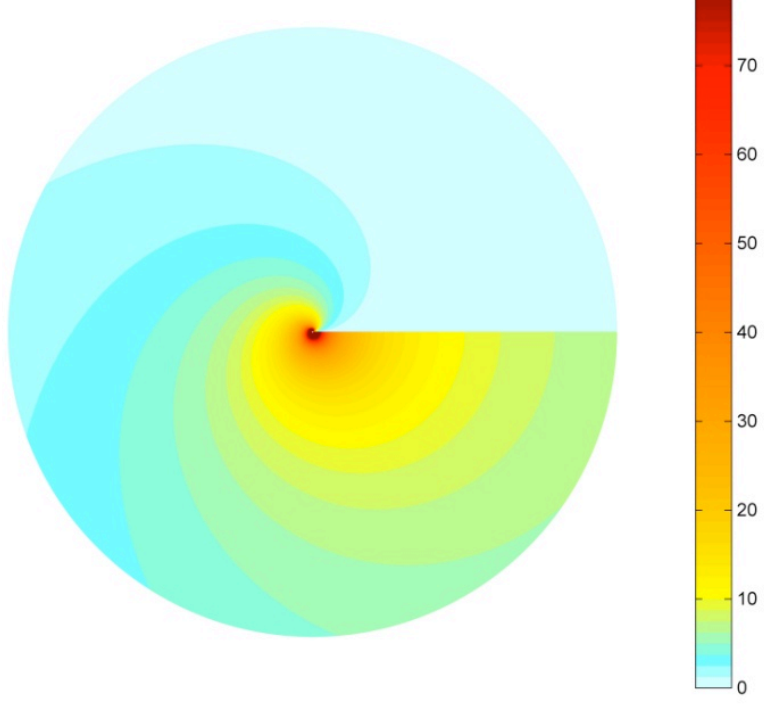

(b) $q_{r}$

Fig.8 Contours of heat flux densities around the tip of the crack shown in Fig.7.

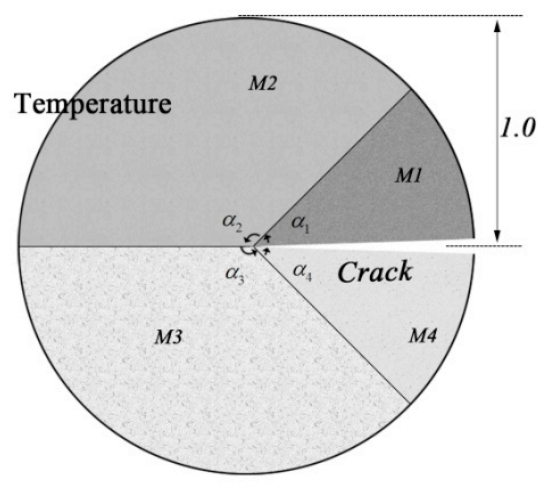

Fig.9 Configuration of a four material disc containing a crack 


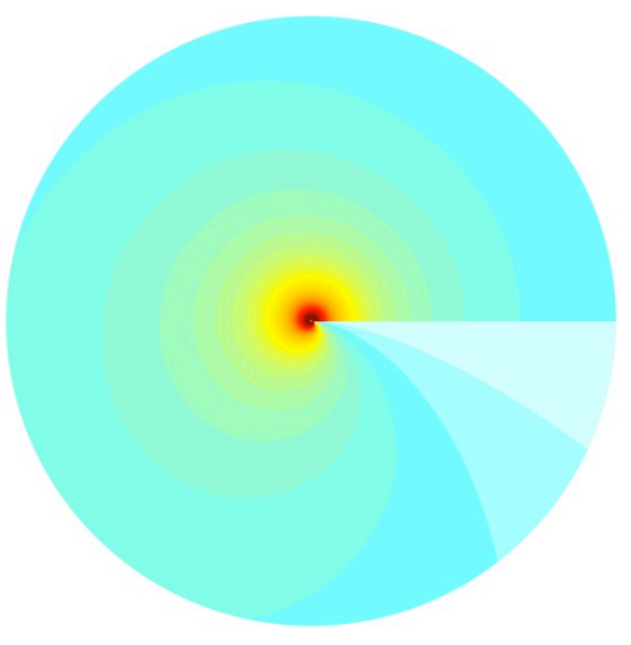

(a) $q_{\theta}$

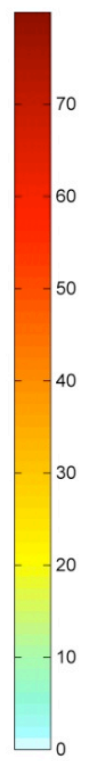

(b) $q_{r}$

Fig.10 Contours of heat flux densities around the four-material crack tip.

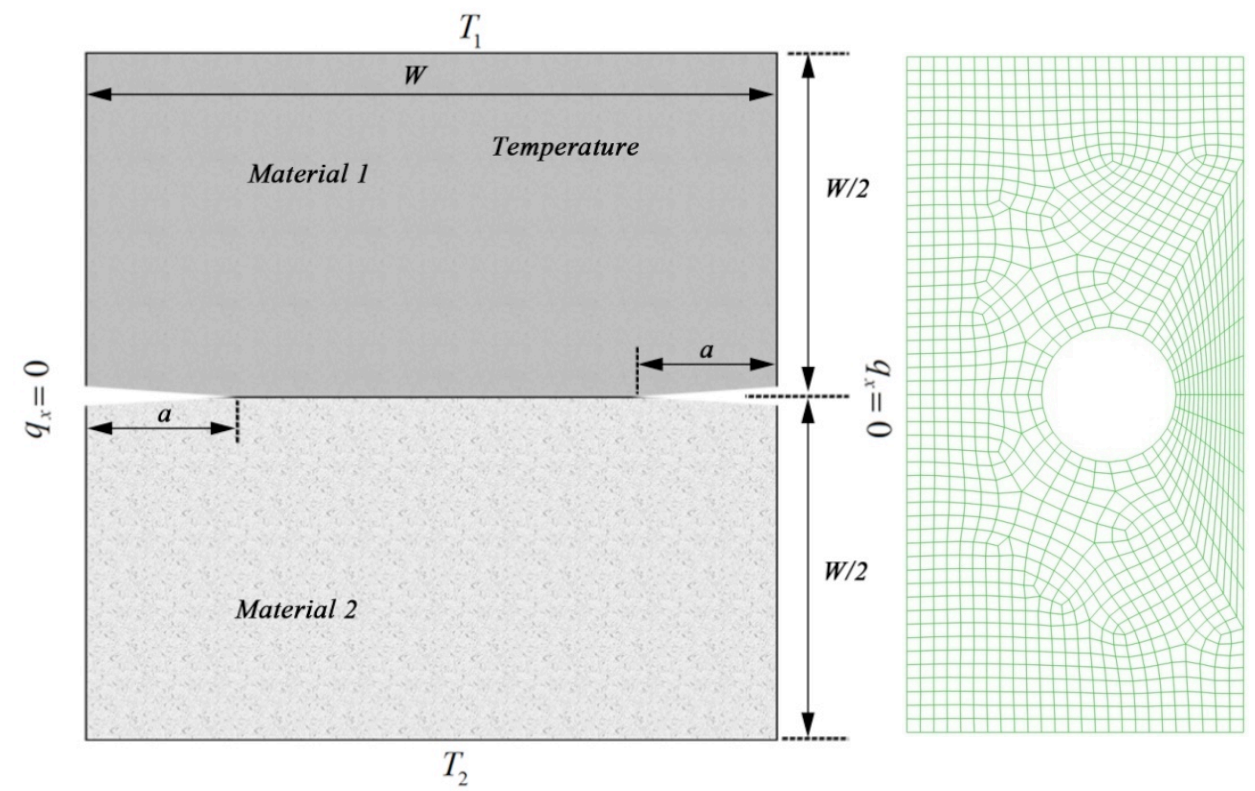

Fig.11 A double edge crack in a bimaterial rectangular plate and the FE mesh 


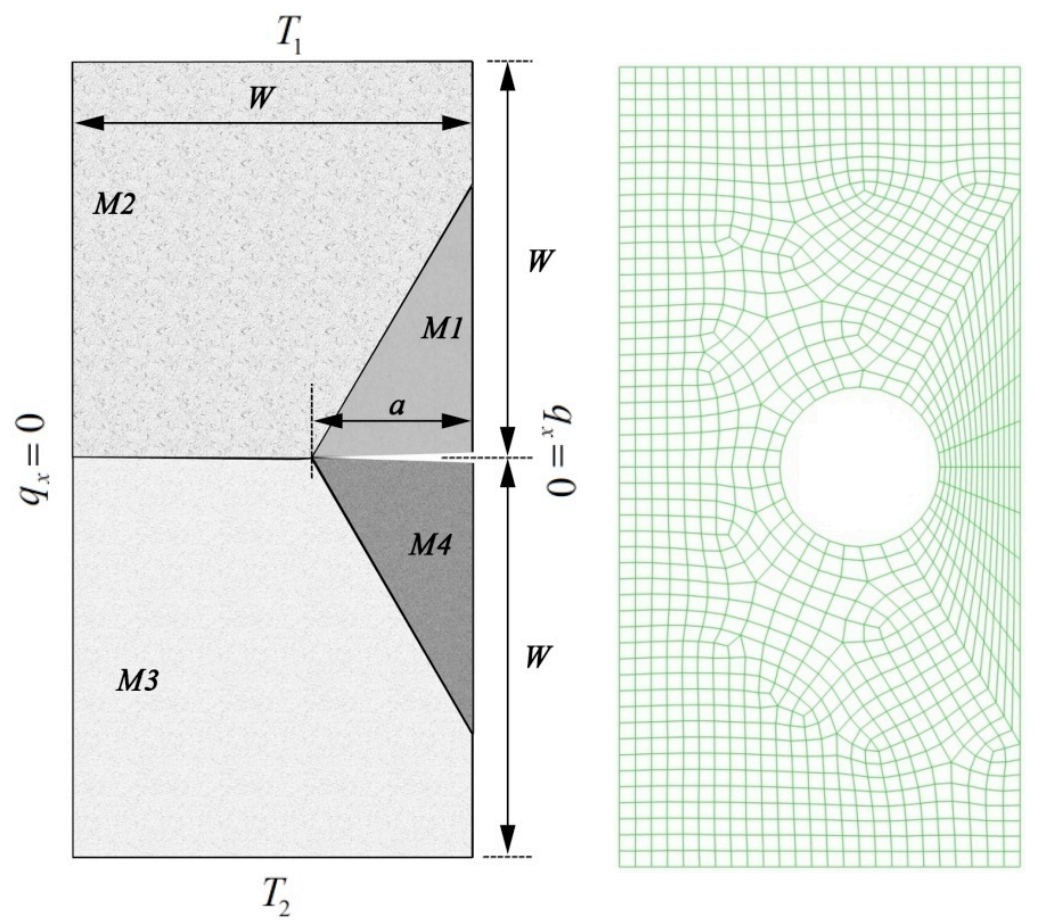

Fig.12 A four material edge crack in a rectangular plate and the FE mesh 
Tab.1 Singularity invariants of the multi-material crack

\begin{tabular}{llllc}
\hline & 7 material & 9 material & 11 material & 13 material \\
\hline$\mu^{\text {invariant } 1}$ & 0.468758 & 0.469982 & 0.470512 & 0.470792 \\
$\mu^{\text {invariant } 2}$ & 0.912260 & 0.929118 & 0.934820 & 0.937516 \\
\hline
\end{tabular}

Tab.2 Generalized flux intensity factors(GFIFs) of the cracked single material disc

\begin{tabular}{cllllll}
\hline Export Node Num. & \multicolumn{1}{c}{$\gamma_{1}$} & Err\% & \multicolumn{1}{c}{$\gamma_{2}$} & Err\% & \multicolumn{1}{c}{$\gamma_{3}$} & Err\% \\
\hline 13 & -1.3361763 & -1.61574 & 0.93698025 & -3.41276 & -0.42601371 & -5.89637 \\
17 & -1.3456556 & -0.91777 & 0.95223888 & -1.83985 & -0.44502567 & -1.69676 \\
31 & -1.3581286 & 0.00063 & 0.97008793 & 0.00009 & -0.45270759 & 0.00013 \\
Ref.[51] & -1.35813 & --- & 0.970087 & --- & -0.452707 & -- \\
Ref.[32] & -1.35812 & --- & 0.970087 & --- & -0.452707 & --- \\
\hline
\end{tabular}

Tab.3 Numerical solutions of the first few symplectic eigenvalues the four material crack

\begin{tabular}{lllllll}
\hline$n=1$ & 2 & 3 & 4 & 5 & 6 & 7 \\
\hline 0.18044 & 0.70620 & 1.17943 & 1.82057 & 2.29380 & 2.81956 & 3.18044 \\
\hline
\end{tabular}

Tab.4 Generalized flux intensity factors(GFIFs) of the cracked four-material disc

\begin{tabular}{clll}
\hline Export Node Num. & $\gamma_{1}$ & $\gamma_{2}$ & $\gamma_{3}$ \\
\hline 13 & -1.09597148 & 0.51025814 & -0.18213196 \\
17 & -1.10293654 & 0.51993674 & -0.19368712 \\
25 & -1.19465521 & 0.52471515 & -0.19978455 \\
31 & -1.19368712 & 0.52227187 & -0.19789354 \\
\hline
\end{tabular}

Tab.5 Generalized flux intensity factors(GFIFs) of the double edge cracked bimaterial plate

\begin{tabular}{llllll}
\hline$T_{2}\left({ }^{\mathrm{o}} \mathrm{C}\right)$ & $\gamma_{1}$ & $\gamma_{2}$ & $\gamma_{3}$ & $\gamma_{4}$ & $\gamma_{5}$ \\
\hline 0 & 15.514225 & 8.751910 & 2.488849 & 0.097302 & -0.027103 \\
120 & 81.575285 & 9.039843 & -0.753853 & 1.156677 & -0.273296 \\
240 & 147.636345 & 9.327776 & -3.996555 & 2.216053 & -0.519490 \\
360 & 213.697405 & 9.615709 & -7.239258 & 3.275428 & -0.765683 \\
\hline
\end{tabular}

Tab.6 Generalized flux intensity factors(GFIFs) of the four material edge crack

\begin{tabular}{llllll}
\hline$T_{2}\left({ }^{\circ} \mathrm{C}\right)$ & $\gamma_{1}$ & $\gamma_{2}$ & $\gamma_{3}$ & $\gamma_{4}$ & $\gamma_{5}$ \\
\hline 0 & 33.887201 & 14.951955 & 2.306122 & 0.098628 & -0.047287 \\
40 & 66.403534 & 14.595335 & 1.082054 & 0.474483 & -0.165561 \\
80 & 98.919866 & 14.238715 & -0.142014 & 0.850339 & -0.283834 \\
120 & 131.436199 & 13.882095 & -1.366082 & 1.226194 & -0.402108 \\
160 & 163.952531 & 13.525475 & -2.590150 & 1.602050 & -0.520382 \\
200 & 196.468864 & 13.168855 & -3.814218 & 1.977906 & -0.638655 \\
\hline
\end{tabular}

